\title{
Calculation algorithm for the dynamic coefficient of vibro-viscosity and other properties of energy willow cuttings movement in terms of their unloading from the tanker
}

\author{
Volodymyr Ivanyshyn ${ }^{1}$, Serhii Yermakov $^{1}$, Tatyana Ishchenko $^{2}$, Krzysztof Mudryk ${ }^{3}$, and \\ Taras Hutsol ${ }^{1, *}$ \\ ${ }^{1}$ State Agrarian and Engineering University in Podilia, 32300 Kamianets-Podilskyi, Ukraine \\ ${ }^{2}$ PO SMC "Ahroosvita", 03151 Kyiv, Ukraine \\ ${ }^{3}$ Faculty of Production and Power Engineering, University of Agriculture in Krakow, Str. Balicka \\ 116b, 30-149 Krakow, Poland
}

\begin{abstract}
When we design the automated systems of unloading materials from the tankers, especially when we construct the automated tool for planting an energy willow, there is a need to create the movement model and to study its characteristics. The combination of cuttings, unloaded from the tanker, can be represented as the flow of a two-phase pseudo liquid. Then the process of unloading itself can be modelled on the basis of methods of hydrodynamic multiphase systems. The main characteristics that affect the process of unloading are highlighted in the deduced equation. The effective coefficient of vibro-viscosity and the calculation algorithm for the dynamic coefficient of vibro-viscosity in terms of cuttings oscillatory motion influenced by gravity field and harmonic vibrations is given in the study.
\end{abstract}

\section{Introduction}

Research on characteristics of materials that are being unloaded from a tanker is an upto-date issue. It will help to get a high-performance selection of material as a result of mechanization and automation of most technological processes. The task of the research is complicated by the fact that each material has certain parameters. For example, when we design the machines for planting energy willow the peculiarities of cuttings flow from the tanker influenced by gravity field should be taken into consideration. $[1,2,6]$. Let us assume that the layer of cuttings consists of circular cylinders with $b$ length, $\bar{\rho}$ density and $a$ radius.

Research on dynamics of bulk material flow from tanks, antibridging strategies and development of bridging equipment was done by the following scientists Alferov K.,

\footnotetext{
*Corresponding author: pro-gp@pdatu.edu.ua
} 
Belousov A., Blehmana I., Gorjushinskyi B. C., Gorjushinskyi I.V., Dzhenike E., Eshutkin D. N., Zheltkov I., Kvapila R., Keglin B. G., Kunakov B. C., Loktionova O. G., Mulkzhina A. P., Semenov V. F., Sokolov V. V., Tretyakov G. M., Ushakov L. N., Jacun S. F. and others. The basic characteristics, physical and mechanical properties of bulk materials that influence the bridging, research on the smooth functioning of bunker devices and development of equipment for bulk cargoes with a wide range of physical and mechanical properties are given in their work.

It should be noted that there is no unified theory in bulk material flow and processes of bridging in the bunker.

The theoretical analysis showed that research on bulk materials flow from tankers is done in the four main lines. The first one is based on the theory of elasticity of continuous medium (G. A. Ganiev); the second line of research is based on the general theory of Coulomb's wedge theory of bulk materials (R. L. Zenkov); the third one is based on granular medium (L. V. Gjachev); the fourth line is the hypothesis of bulk materials bridging, i.e. the ability of a loose body to form an arched structure of discrete particles (V.A. Nadezhdyn, V. A. Bohomiagkih) [3-5, 12, 16-18].

\section{Materials and methods}

The theoretical basis of the study is the researches scientists on scientific methods of bulk material unloading from containers, issues of bridging problems and continuous flow of material.

As a model for quickset unloading bunker we consider two half-planes that are located at $\alpha$ and $\beta$ angles according to the horizontal plane. The width of the unloaded window can be identified as $b$ (fig.1). Let us introduce the Cartesian coordinate system $x_{1}, x_{2}, x_{3}$ with the axis $x_{3}$ that coincides with the line passing through the center of the unloaded window.

To model the quickset movement in the middle of the bunker methods of hydrodynamics of multiphase systems is used. According to this approach, the combination of quickset is considered to be pseudoliquid that consists of two phases: a discrete phase formed by cuttings and continuous phase formed by gaseous medium (air). Each of these phases is regarded to be medium that allowed us to consider uploading as the movement of viscous incompressible pseudoliquid. The velocity speed of this pseudoliquid must satisfy Navier-Stokes equation. At the same time reviewed such characteristics of a two-phase pseudo-liquid as the density and viscosity of each of the phases.

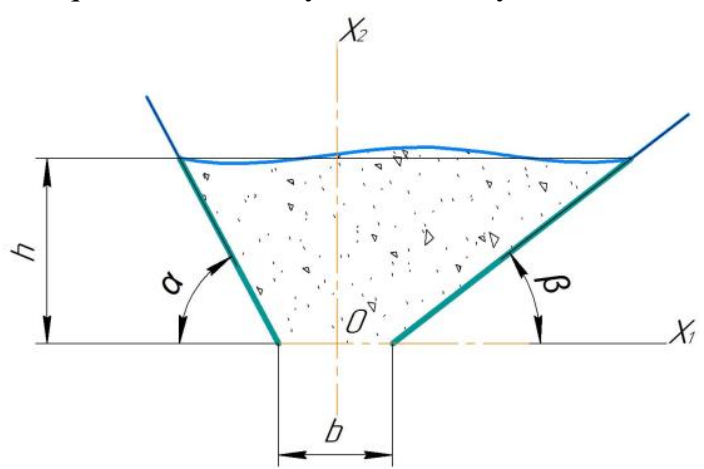

Fig. 1. Design scheme of the bunker for cuttings 
The algorithm for determining these characteristics, the coefficient of vibro-viscosity, in particular, is developed by analogy with results obtained previously by L.N. Tishhenko (he examined the intensification of grain separation in terms of unloading from the tanker) $[7 ; 8]$.

\section{Results and discussion}

The combination of cuttings that are going to be unloaded can be represented as a structure that consists of two components, where the cuttings are a discrete component. And gaseous medium (air) fills all the rest of space. The process of unloading of such a structure from a tanker can be modelled on the basis of methods of hydrodynamic multiphase systems $[9 ; 10]$. According to this approach, we believe that the combination of cuttings that is affected by the gravitational field and the vibrating motions can be observed as the pseudo liquid. This liquid consists of two phases: a discrete phase that is formed by cuttings and continuous phase - gaseous medium (air). Each of these phases from the mechanic multiphase systems point of view is represented as solid mediums with certain characteristics. So for each phase, we can offer the parameter of density: $\rho$ - for discrete phases, and $\rho_{1}$ - for continuous phase. These options will depend on the average density of its parts ( $\bar{\rho}$ - cuttings, $\bar{\rho}_{1}$ - air, and their bulk concentration).

In addition to $\rho$ and $\rho_{1}$ parameters, the two-phase pseudo liquid is characterized by two effective dynamic coefficients of vibro-viscosity that are determined by both the interaction between cuttings and cuttings with gaseous medium (air). Let us take $\mu$ for a coefficient of vibro-viscosity of discrete phases that are determined by the interaction between cuttings and $\mu_{1}$ for a dynamic viscosity coefficient of gaseous (air) medium.

Let's assume that the bulk concentration of cuttings (discrete phase) is much bigger than a similar concentration of cuttings of the continuous phase. In this case, the effective coefficient of vibro-viscosity that is determined by the interaction between cuttings and gaseous medium can be ignored.

Therefore, the movement of discrete components (the total of cuttings) can be modelled as a movement of viscous incompressible pseudo liquid. Velocity field of this pseudo liquid satisfies the Navier-Stokes equation and the solution of this equation in a linear approximation will be a mathematical model of unloading the cuttings from the tanker. To derive the equations of motion of a two-phase velocity field let us present the velocity speed as $\vec{V}$ - velocity fields of a discrete phase and $\vec{V}_{1}$ - velocity fields of the continuous phase. In accordance with [11], the equations of motion of a two-phase pseudo liquid can be represented in the following form

$$
\begin{aligned}
& \rho\left(\frac{\partial \vec{V}}{\partial t}+\left(\vec{V}_{1}, \nabla\right) \cdot \vec{V}\right)=-\nabla P+\mu \Delta \vec{V}+\vec{F}-\rho g e_{2}, \\
& \operatorname{div} \vec{V}=0
\end{aligned}
$$

Here $e_{1}, e_{2}, e_{3}$ are unit vectors of the Cartesian coordinate system, $P$ - are pressures of a discrete phase, $g$ is free fall acceleration, $\vec{F}$ is the mass force that acts on the mass 
unit of discrete components of the pseudo liquid. As S. Sow works show [11], $\vec{F}$ can be represented as:

$$
\vec{F}=0,5 \frac{\bar{\rho}_{1}}{\bar{\rho}} \frac{\partial}{\partial t}\left(\vec{V}_{1}-\vec{V}\right)+\frac{9 \bar{\rho}_{1} \sqrt{\nu}}{2 \sqrt{\pi} a \bar{\rho}} \int_{0}^{t}(t-\tau)^{-1 / 2} \frac{d\left(\vec{V}_{1}-\vec{V}\right)}{d t} d \tau+\vec{f}
$$

Where $\bar{\rho}_{1}$ and $v_{1}$ are the density and kinematic viscosity coefficient of continuous phase; $\frac{d}{d t}$ is total time derivative, $a$ is the radius of the circle on the square that coincides with an average area of cross-sections of cuttings.

Equations (1) and (2) are nonlinear integrodifferential. It is possible to solve equations only with the help of a computer numerical methods [13]. In addition, as it follows from (1), you should enter such important characteristic as an effective coefficient of vibroviscosity $(\mu)$ in terms of, hydrodynamic modelling of cuttings movement. To define this coefficient is an important and complex task because its solution enables you to evaluate the adequacy of mathematical models to physical processes that accompany the cuttings movement from the tanker $[7 ; 8]$.

The task of defining a dynamic vibro-viscosity is based on equation (1) and a number of assumptions. Let layer cuttings be on the horizontal plane. This plane coincides with $x_{1} x_{3}$ plane of the Cartesian coordinate system. The $x_{2}$ axis is directed vertically upwards.

Let us assume that the plane commits harmonic vibrations that are parallel to the $x_{2}$ axis of $A$ amplitude and circular frequency. We suppose that a layer of cuttings consists of circular cylinders with $\bar{\rho}$ density and $a$ radius. A layer of cuttings commits an oscillating motion along $x_{2}$ axis influences by a gravitational field and harmonic oscillation. We will consider the movement of this layer to be the solid movement of the medium (pseudo liquid with $\rho$ density and $\mu$ dynamic coefficient of vibro-viscosity. Density $\rho$ is connected with $\bar{\rho}$ density by the formula [11]

$$
\rho=\bar{\rho} \delta \text {, }
$$

where $\delta$-volume concentration of cuttings in the layer.

Under the influence of a gravitational field and vibration hesitation, A layer of pseudo liquid is influenced by the gravitational field and vibration hesitation and moves along $x_{2}$ the axis at a rate that depends only on temporary variable according to the formula

$$
V=A \omega \sin \omega t \text {. }
$$

Let us consider the motion of a single cutting in such pseudo liquid. The velocity vector of the seedlings will be parallel to $x_{2}$ axis and must satisfy the equation (1). Taking into account the assumptions this equation will take the form of:

$$
\begin{aligned}
& \rho_{1} \frac{\partial V_{1}}{\partial t}=\frac{\rho_{1} \bar{\rho}}{2 \bar{\rho}_{1}} \frac{\partial\left(V-V_{1}\right)}{\partial t}+\frac{9 \bar{\rho} \rho_{1} \sqrt{v}}{2 \sqrt{\pi} a \bar{\rho}_{1}} \int_{0}^{t} \frac{\partial\left(V-V_{1}\right)}{\partial \tau}(t-\tau)^{-1 / 2} d \tau+ \\
& \frac{75 \bar{\rho} \rho_{1} v \delta_{1}}{2 a^{2} \bar{\rho}_{1}\left(1-\delta_{1}\right)^{2}}\left(V-V_{1}\right)-\rho_{1} g .
\end{aligned}
$$


Here $V_{1}$ is the velocity of the sapling, $v=\mu / \rho$ is a kinematic coefficient of vibroviscosity of pseudo liquid, $a$ is the radius of the cylinder, that models a seedling, $\rho_{1}=\bar{\rho} \delta_{1}, \bar{\rho}_{1}=\bar{\rho}, \delta_{1}$ is volume concentration of seedlings in pseudo liquid, $\delta_{1}=1-\delta$.

An integral member in (6) is determined by Boss power and identifies the instantaneous moving resistance of seedlings by pseudoliquid. Subsequently, we will ignore this member because the value of $A$ amplitude is fairly small, and relevant time of speed changes in pseudo liquid $T=\frac{2 \pi}{\omega}$ is high. In this case, the equation (6) is easier and takes the following form

$$
1.5 \frac{\partial u}{\partial t}+\Phi u=g+A u^{2} \cos \omega t
$$

Where $u=V-V_{1}$ is the fractional speed of the seedling,

$$
\Phi=\frac{75 v(1-\delta)}{2 \delta^{2} a^{2}}
$$

Identifying $u$ (relative velocity of seedlings) from (7) can be done with the help of Laplace transforms [8]. Let $\bar{u}$ determines Laplace transforms according to $u$ speed

$$
\bar{u}=\int_{0}^{\infty} u(t) e^{-q t} d t
$$

Let us apply Laplace transform to the left and right parts (7). Then we'll have

$$
\bar{u}(1.5 q+\Phi)=\frac{g}{q}+\frac{A \omega^{2} q}{\omega^{2}+q^{2}} \text {. }
$$

We have the following from (10)

$$
\bar{u}=(1.5 q+\Phi)^{-1}\left(\frac{g}{q}+\frac{A \omega^{2} q}{\omega^{2}+q^{2}}\right) \text {. }
$$

When we apply to (11) the transformation that is inverse to Laplace transformation, we obtain

$$
u(t)=\frac{1}{2 \pi i} \int_{c-i \infty}^{c+i \infty}(1.5 q+\Phi)^{-1}\left(\frac{g}{q}+\frac{A \omega^{2} q}{\omega^{2}+q^{2}}\right) e^{q t} d q
$$

where $c>0$.

To calculate the integral (12) we will use the method of deduction [9]. The integrand function in(12), being a function of the complex $q$ variable is analytical and has characteristics of a simple pole type at $q=0,-\frac{2}{3} \Phi, \pm i \omega$. 
In addition, when $\operatorname{Re} q<0$ and $\operatorname{Re} q \rightarrow-\infty$, this function tends to zero $(\operatorname{Re}(\ldots) u \operatorname{Im}(.$.$) denote real and imaginary parts of a complex number). These$ properties of integrand function allow us to apply the theorem of residues [9] and replace the integral (11) in the number of deductions

$$
\begin{aligned}
& \frac{1}{2 \pi i} \int_{c-i \infty}^{c+i \infty}(1.5 q+\Phi)^{-1}\left(\frac{g}{q}+\frac{A \omega^{2} q}{\omega^{2}+q^{2}}\right) e^{q t} d q=\operatorname{Re} s(0)+\operatorname{Re} s(i \omega)+ \\
& +\operatorname{Re} s(-i \omega)+\operatorname{Re} s\left(-\frac{2}{3} \Phi\right)
\end{aligned}
$$

Having calculated the deductions we have

$$
u(t)=\frac{g}{\Phi}-\left(\frac{g}{\Phi}+\frac{4 \Phi A \omega^{2}}{9\left(\omega^{2}+\frac{4}{9} \Phi^{2}\right)}\right) e^{-\frac{2}{3} \Phi t}+A \omega^{2} \operatorname{Re}\left(\frac{e^{i \omega t}}{\Phi+1.5 \omega i}\right)
$$

We'll focus on the steady process of seedling movement of seedlings in pseudo liquid. Then the second member in (14) can be ignored and finally get the formula for the relative speed of the seedling.

$$
u(t)=\frac{g}{\Phi}+A \omega^{2} \frac{\Phi \cos \omega t+1.5 \omega \sin \omega t}{\Phi^{2}+2.25 \omega^{2}} .
$$

We use (15) in order to obtain an equation that groups together the dynamic coefficient of vibro-viscosity and physics and mechanics characteristics of the cuttings. Then we apply the equivalence principle of pulse resistance forces to cutting movement in different mediums [10]. A similar approach was applied in [7].

Let's calculate the impulse of resistance force to seedlings movement for one halfperiod of pseudoliquid fluctuations. In accordance with the equation (7) we'll present the resistance force in the form of

$$
\Phi_{c}=\pi a^{2} l \rho_{1} \Phi u
$$

where $l$ is the length of the seedlings (average for all the seedlings), $\rho_{1}=\bar{\rho} \delta_{1}$ reduced density. Coefficient $\Phi$ is determined by the formula (8). On the basis of (11), we have:

$$
I_{c}=\pi a^{2} l \rho_{1} \Phi \int_{0}^{\pi / \omega} u d t=\pi a^{2} l \rho_{1} \Phi\left(\frac{g \pi}{\Phi \omega}+\frac{3 A \omega^{2}}{\Phi^{2}+2.25 \omega^{2}}\right)
$$

On the other hand, the pseudo liquid is a collection of cuttings and friction influences the movement of separate seedling, which can be represented as

$$
\Phi_{T}=f \bar{\rho} g\left(h-x_{2}\right) s
$$

Where $f$ is the coefficient of friction between the plants, $g$ is the gravitational acceleration, $h$ is the thickness of the cuttings, $s$ is midsection of a sapling, $x_{2}$ is 
coordinate along the vertical axis that defines the location of seedlings according to the horizontal plane.

From (18) we get

$$
\Phi_{T}=f \bar{\rho} g\left(h-x_{2}\right) 2 a l \text {. }
$$

In this case, the impulse of force $\Phi_{T}$ is

$$
I_{T}=\frac{\pi}{\omega} 2 \operatorname{alf} \bar{\rho} g\left(h-x_{2}\right)
$$

Comparing $I_{c}$ and $I_{T}$ we have the equation for finding the dynamic coefficient of vibro-viscosity

$$
\frac{\Phi}{\Phi^{2}+2.25 \omega^{2}}=\bar{A}
$$

where

$$
\bar{A}=\frac{g\left(2 f\left(h-x_{2}\right)-\pi a \delta_{1}\right)}{A \omega^{3} a \delta_{1}},
$$

Value $\Phi$ is expressed through the dynamic coefficient of vibro-viscosity according to the formula (8).

The ratio of (21) is the equation of relative magnitude $\Phi$. If $\Phi_{0}$ is equation root (21), as can be seen from (8), the dynamic coefficient of vibro-viscosity is

$$
\mu=\frac{2 \Phi_{0} \bar{\rho} \delta^{3} a^{2}}{75(1-\delta)}
$$

Consequently, to determine $\mu$ it is enough to find the root $\Phi_{0}$ of equation (21). It is easy to notice that equation (21) has real roots only if there is inequality

$$
0 \leq \bar{A} \leq \frac{1}{3 \omega}
$$

Next, let this inequality be completed. We have with the help of (21)

$$
\Phi_{0}=\frac{1 \pm \sqrt{1-9 \bar{A}^{2} \omega^{2}}}{2 \bar{A}} \text {. }
$$

From two roots (25) we choose the one that in terms of $\bar{A} \rightarrow 0$ tends to zero.

$$
\Phi_{0}=\frac{1-\sqrt{1-9 \bar{A}^{2} \omega^{2}}}{2 \bar{A}}=\frac{9 \bar{A} \omega^{2}}{2\left(1+\sqrt{1-9 \bar{A}^{2} \omega^{2}}\right)} \text {. }
$$

We will substitute (26) to (23) and get

$$
\mu=\frac{3 \bar{A} \omega^{2} \bar{\rho} \delta^{3} a^{2}}{25(1-\delta)\left(1+\sqrt{1-9 \bar{A}^{2} \omega^{2}}\right)}
$$


Formula (27) is just when we complete inequality (24). In addition, the thickness of the cuttings $h$ should satisfy the inequality (it follows from (24))

$$
h>\frac{\pi a(1-\delta)}{2 f} \text {. }
$$

As can be seen from (22), the dynamic coefficient of vibro-viscosity depends on the spatial variable. In order to simplify the theory, we will continue to use the averaged value $\mu_{c p}$. Its finding is associated with the computing the value of integrals

$$
\begin{aligned}
& \mu_{c p}=\frac{1}{\bar{h}} \int_{0}^{\bar{h}} \mu d x_{2} \\
& \text { where } \bar{h}=\frac{\pi a(1-\delta)}{2 f} .
\end{aligned}
$$

Let us substitute (17), (22) for (29). And we arrive at the formula

$$
\mu_{c p}=\frac{A \omega^{3} a^{3} \delta^{3} \bar{\rho}}{150 \bar{h} g f}\left(\frac{x_{0}}{1+\sqrt{1-x_{0}}}-\ln \frac{2}{1+\sqrt{1-x_{0}}}\right)
$$

where

$$
x_{0}=\frac{36 g^{2} f^{2}(h-\bar{h})^{2}}{A^{2} \omega^{4} a^{2}(1-\delta)^{2}} .
$$

As a result, we received formula for calculating the dynamic coefficient for vibroviscosity of cuttings layer that is in oscillating motion and is influenced by a gravitational field and harmonic vibrations.

\section{Conclusions}

1. When we create a variety of automated systems for material feed there is a need to ensure high performance of unloading and selection of material. In particular, we face this problem in energy willow planting machine design.

2. The combination of energy willow cuttings that are going to be unloaded can be represented as a viscous pseudo liquid that consists of two phases: a discrete phase formed by cuttings and the continuous phase (gaseous medium (air)). Each of these phases in terms of the mechanics of multiphase systems are represented as solid mediums with certain characteristics. According to these assumptions, the process of unloading can be modelled on the basis of methods of hydrodynamics of multiphase systems. In addition, the field speeds of such pseudo liquid must satisfy the NavierStokes equation.

3. According to the equation, it is important to know such characteristics of pseudo liquid as density, the viscosity of its components to get the motion model of cuttings.

4. It is a very important and difficult task to determine the effective coefficient of vibroviscosity $\mu$, because it enables us to evaluate the adequacy of mathematical models of physical processes that accompany the seedlings movement of in the tanker. 
5. The algorithm for determining the coefficient of vibro-viscosity of cuttings by analogy with results obtained by L. N. Tyshchenko in the research study of intensification of grain separation.

6. The coefficient of vibro-viscosity depends on the amplitude and frequency of vibrations, the acceleration of free fall, geometric parameters and the average density of cuttings and the dry friction coefficient between the plants.

\section{References}

1. S.V. Yermakov Perspektyvy udoskonalennia konstruktsii dlia sadinniazhyvtsiv enerhetychnykh kultur [Perspectives of improvement of constructions for energy crop planting]. Podilian Bulletin: agriculture, engineering, economics, v. 2, n. 26, p. 37-45. (2017)

2. T. Hutsol, S. Yermakov, Ju. Firman, V. Duganets, A. Bodnar Analysis of technical solutions of planting machines, which can be used in planting energy willow.

Renewable Energy Sources: Engineering, Technology, Innovation. ICORES 2018 (to be published)

3. V.A. Bohomiahkih, A.P. Pepchuk Intensifikaciya razgruzki bunkernyx ustrojstv v usloviyax svodoobrazovaniya zernistyx materialov [The improvement of unloading of hopper devices in terms of grain bridging]. Zernohrad. (1985)

4. L.V. Gyachev Osnovy teorii bunkerov [Fundamentals of hopper theory]. Novosibirsk: izd-vo novosibirskogo universiteta. 184p. (1992)

5. R.L. Zenkov, G.P. Grinevich, V.S. Isaev Bunkernye ustrojstva [Hopper devices]. Moskow: Mashinostroenie. 224p. (1966)

6. S. Yermakov, T. Hutsol, S. Slobodian, S. Komarnitskyi, M. Tysh Possibility of using automation tools for planting of the energy willow cuttings. Renewable Energy Sources: Engineering, Technology, Innovation. ICORES 2018 (to be published)

7. L.N Tyshchenko Intenifikaciya separirovaniya zerna [Identification of grain separation]. Kharkov: Osnova, 224 p. (2004)

8. L.N. Tyshchenko, V.N. Olshanskyi Vibroreshyotochnaya separaciya zernovyx smesej. [Vibro latticed separation of grain mixes]. Kharkov: Misruk. 280p. (2011).

9. M.A. Lavrentev, B.V. Shabat Metody teorii funkcij kompleksnogo peremennogo [Methods of the theory of functions of a complex variable. Moskow: Izd-vo Physics and Mathematics Lit. 647p. (1958)

10. N.N. Boholiubov, J.A. Mytropolskyi Asimptoticheskie metody v teorii nelinejnyx kolebanij [Asymptotic methods in the theory of nonlinear oscillations]. Moscow: Nauka. 504p. (1974)

11. S. Sous Gidrodinamika mnogofaznyx sistem [Hydrodynamics of multiphase systems]. Moscow: Mir. 536p. (1971).

12. S. Yermakov, T. Hutsol Features of the heterogeneous rod-like materials outflow. Technological and methodological aspects of agri-food engineering in young scientist research. Krakow, 2018, pp. 55-68 (2018)

13. R.I. Nyhmatulin Osnovy mexaniki geterogennyx sred [The basics of mechanics of heterogeneous mediums]. Moscow: Nauka. 336p. (1978)

14. Y. Pantsyr, I. Garasymchuk, T. Hutsol, Gordiychuk I. Energy Parameters' Calculation of a Hybrid Heat Supply System for a Private House in the Conditions of Western Part of Ukraine. Renewable Energy Sources: Engineering, Technology, Innovation. Springer, Cham. 2018, pp. 765-780. (2018) 
15. K. Dziedzic, K. Mudryk, T. Hutsol, B Dziedzic Impact of grinding coconut shell and agglomeration pressure on quality parameters of briquette. Engineering for rural development. Jelgava, 23.-25.05.2018. pp.1884-1889 (2018)

16. S. Yermakov, T. Hutsol, O. Ovcharuk, I. Kolosiuk Mathematic simulation of cutting unloading from the bunker. Independent journal of management \& amp; production (IJM\&P). Pp. 758-777 (2019)

17. S. Yermakov, T. Hutsol Features of the heterogeneous rod-like materials outflow. Technological and methodological aspects of agri-food engineering in young scientist research. Krakow, p. 55-68. (2018)

18. S. Yermakov, K. Mudryk, T. Hutsol, K. Dziedzic, L. Mykhailova The analysis of stochastic processes in unloadingthe energywillow cuttings from the hopper. Environment. Technology. Resources. Rezekne, Latvia. Proceedings of the 12th International Scientific and Practical Conference (to be published)

19. K. Dziedzic, B. Lapczyńska-Kordon, K. Mudryk (2017) Decision support systems to establish plantations of energy crops on the example of willow (Salix Viminalis L.). Scientific achievements in agricultural engineering, agronomy and veterinary medicine polish Ukrainian cooperation, v. 1, n. 1, p. 150-160. 